\title{
PHLDA3 inhibition attenuates endoplasmic reticulum stress-induced apoptosis in myocardial hypoxia/reoxygenation injury by activating the PI3K/AKT signaling pathway
}

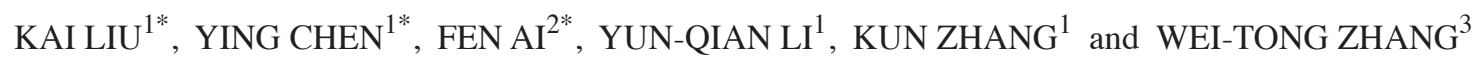 \\ ${ }^{1}$ Department of Geriatric Center, Hainan General Hospital, Hainan Affiliated Hospital of Hainan Medical University, \\ Haikou, Hainan 570311; ${ }^{2}$ Department of Emergency, The Central Hospital of Wuhan, Tongji Medical College, \\ Hua Zhong University of Science and Technology, Wuhan, Hubei 430000; ${ }^{3}$ Department of General Surgery, \\ Hainan General Hospital, Hainan Affiliated Hospital of Hainan Medical University, Haikou, Hainan 570311, P.R. China
}

Received March 31, 2020; Accepted September 24, 2020

DOI: 10.3892/etm.2021.10045

\begin{abstract}
Endoplasmic reticulum stress (ERS)-induced apoptosis serves a crucial role in the pathogenesis of myocardial ischemia/reperfusion injury (MIRI). Previous studies have confirmed that pleckstrin homology-like domain family A member 3 (PHLDA3) is an important mediator in ERS-associated apoptosis. The aim of the current study focused on whether PHLDA3 served protective effects on hypoxia/reoxygenation (H/R)-injured cardiomyocytes by inhibiting ERS-induced apoptosis. Furthermore, the molecular mechanisms associated with the PI3K/AKT signaling pathway were investigated. Primary neonatal rat cardiomyocytes were isolated and randomized into four groups: i) Control + adenovirus encoding scrambled short hairpin RNA (AdshRNA); ii) control + adenoviral vectors encoding PHLDA3 shRNA (AdshPHLDA3); iii) H/R+ AdshRNA and iv) $\mathrm{H} / \mathrm{R}+\mathrm{AdshPHLDA} 3$. AdshPHLDA3 was used to knock down PHLDA3. An H/R injury model was constructed by treatment with hypoxia for $4 \mathrm{~h}$ followed by reoxygenation for 6 h. A PI3K/AKT inhibitor, LY294002, was supplemented in mechanistic studies. Cell viability and LDH/CK releases were detected to evaluate myocardial damage. Flow cytometry assays were used to assess apoptotic response. Western blotting assays were used to detect protein expression. The results demonstrated that $\mathrm{H} / \mathrm{R}$ induced myocardial damage
\end{abstract}

Correspondence to: Dr Wei-Tong Zhang, Department of General Surgery, Hainan General Hospital, Hainan Affiliated Hospital of Hainan Medical University, 19 Xiuhua, Haikou, Hainan 570311, P.R. China

E-mail: liugeriatric@163.com

${ }^{*}$ Contributed equally

Key words: pleckstrin homology-like domain family A member 3, hypoxia/reoxygenation, endoplasmic reticulum stress, apoptosis, PI3K/AKT and increased PHLDA3 expression. ERS-induced apoptosis was significantly increased following H/R injury, as indicated by increased apoptotic rates and ERS-associated protein expression, including those of CHOP, $78 \mathrm{kDa}$ glucose-regulated protein and caspase-12. However, PHLDA3 inhibition following AdshPHLDA3 transfection reversed cell damage and ERS-associated apoptosis on H/R injury. Studies for molecular mechanisms concluded that the apoptosis-inhibition effects and cardioprotective roles of PHLDA3 inhibition were induced partly by the activation of the PI3K/AKT pathway, which was verified by LY294002 treatment. In conclusion, in the process of $\mathrm{H} / \mathrm{R}$ injury, PHLDA3 inhibition reduced ERS-induced apoptosis and H/R injury by activating the PI3K/AKT pathway. PHLDA3 may be a therapeutic target for the treatment of MIRI.

\section{Introduction}

The most effective intervention for acute myocardial infarction is timely revascularization (1-3). However, re-establishing blood to ischemic areas yields additional myocardial damage including inflammation, apoptosis and autophagy, which is known as myocardial ischemia/reperfusion (I/R) injury (MIRI) (1-3). The pathophysiological mechanism of MIRI is complex and numerous studies have confirmed that endoplasmic reticulum (ER) stress (ERS)-induced apoptosis serves important roles in the progression of MIRI $(4,5)$. Conventionally, the ER is indispensable for the biosynthesis, folding, processing, excretion and transportation of proteins $(4,5)$. However, the disruption of ER homeostasis caused by MIRI, which is referred to as ERS, leads to inaccurate synthesis and assembly of proteins $(4,5)$. Increasing evidence has indicated that the activation of ERS and the consequent elevation of apoptotic cascade events act as crucial pathogenic mechanisms in MIRI (4,5). Targeting ERS-associated apoptosis may be an important therapeutic approach for MIRI.

Pleckstrin homology-like domain family A member 3 (PHLDA3) serves as a crucial member of the PHLDA family and encodes a small, 127-amino acid protein containing only a PH domain (6-8). PHLDA3 was reported to be highly 
associated with various physiological conditions, including pressure overload-induced cardiac remodeling (9), vascular development (6) and tumor development (8). Among these, the PHLDA3-mediated apoptotic cascade is hypothesized to be a common pathological mechanism in a wide range of diseases, including hepatocyte injury and cardiac remodeling $(7,10)$. Han et al (7) demonstrated that PHLDA3 was upregulated in liver injury with ERS induction and that PHLDA3 overexpression was associated with the severity of hepatocyte injury in a cell model of ERS. However, the roles of PHLDA3 in myocardial I/R injury associated with ERS and apoptosis remain unknown.

Stimulation of the p-PI3K/AKT signaling pathway serves a central role in the protection of MIRI $(4,11)$. The repression of ERS-induced apoptosis resulting from $\mathrm{p}-\mathrm{PI} 3 \mathrm{~K} / \mathrm{AKT}$ activation was verified as a therapeutic avenue in cardioprotection during the initiation and development of MIRI $(11,12)$. Notably, numerous studies reported that PHLDA3 is a negative modulator in multiple pathological disorders, including vascular development, cardiac hypertrophy and islets engraftment, mainly by its inhibition of the p-AKT pathway $(6,9,13)$. However, whether repression of the p-PI3K/AKT pathway could also be modulated via PHLDA3 in MIRI remains unknown. The aim of the current study was to determine whether PHLDA3 served protective effects on hypoxia/reoxygenation (H/R)-injured cardiomyocytes by inhibiting ERS-induced apoptosis.

\section{Materials and methods}

Chemicals and reagents. DMEM, PBS, trypsin, FBS and collagenase type II were purchased from Gibco; Thermo Fisher Scientific, Inc. Cell Counting Kit-8 (CCK-8) and LY294002 (a p-PI3K/AKT inhibitor) were purchased from Dojindo Molecular Technologies, Inc. and Selleck Chemicals, respectively. Lactate dehydrogenase (LDH; cat. no. 03010703011) and creatine kinase (CK; cat. no. 03010702011) were detected by commercially available ELISA kits (Nanjing Jiancheng Bioengineering Institute). The following primary antibodies were obtained from Abcam: PHLDA3 (cat. no. ab22822; 1:500), phosphorylated (p)-PI3K (cat. no. ab182651; 1:400), PI3K (cat. no. ab180967; 1:300), 78 kDa glucose-regulated protein (GRP78; cat. no. ab108615; 1:600), cleaved caspase-12 (cat. no. ab62484; cat. no. 1:600) and GAPDH (cat. no. ab8245, 1:500). Antibodies against p-AKT (cat. no. 4060; 1:600), AKT (cat. no. 4691; 1:400) and CHOP (cat. no. 5554; 1:600) were purchased from Cell Signaling Technology, Inc. Horseradish peroxidase-conjugated goat-anti rabbit secondary antibodies were obtained from BIOSS. A BCA protein assay kit was purchased from Pierce, Thermo Fisher Scientific, Inc.

Neonatal rat cardiomyocyte (NRCM) culture. NRCMs were isolated from the ventricles of 144 1-3-day-old male Sprague-Dawley rats (body weight, 15-20 g) as previously described (9,14-16). Animals were purchased from the Animal Experimental Center of Hainan Medical University (Haikou, China). Animals were bred in a standard environment with controlled temperature $\left(20-25^{\circ} \mathrm{C}\right)$, humidity $(40-60 \%)$ and light conditions (12 h light/dark cycles). Experiments and animal care were performed in adherence with the Guide for the Care and Use of Laboratory Animals published by the US
National Institutes of Health (NIH; 8th Edition; 2011) (17) and were approved by the Hainan Affiliated Hospital of Hainan Medical University.

Cardiomyocyte isolation was performed as previously described (17). Briefly, rat hearts were quickly removed following sacrifice by swift decapitation according to the Guide for the Care and Use of Laboratory Animals published by the US NIH (17). Large blood vessels were excised carefully, and the obtained ventricles were rinsed in ice-cold PBS three times to remove residual blood. Following this, ventricles were placed in a dish with ice-cold PBS. Heart tissues were digested with $0.08 \%$ collagenase type II and $0.125 \%$ trypsin at room temperature for $5 \mathrm{~min}$. NRCMs were centrifuged at $110 \mathrm{x}$ g for 10 min at $37^{\circ} \mathrm{C}$ and resuspended in DMEM supplemented with $10 \% \mathrm{FBS}$ and $1 \%$ penicillin/streptomycin (HyClone; Cytiva) in a humidified incubator at $37^{\circ} \mathrm{C}$ with $5 \% \mathrm{CO}_{2}$ and $95 \% \mathrm{O}_{2}$.

Construction of a hypoxia/reoxygenation $(H / R)$ injury model and experimental groups. H/R injury was established as previously described (14). Briefly, cultured NRCMs were washed twice with PBS and preserved in serum-free DMEM. Cells were then incubated at $37^{\circ} \mathrm{C}$ in an anaerobic chamber for $4 \mathrm{~h}$. Following this, cells were transferred to a normal incubator with $95 \% \mathrm{O}_{2}$ and $5 \% \mathrm{CO}_{2}$ at $37^{\circ} \mathrm{C}$ for an additional $6 \mathrm{~h}$ for reoxygenation.

To determine the possible function of PHLDA3 in MIRI, primary cardiomyocytes were randomly divided into four groups: i) Control + adenovirus encoding scrambled short hairpin (Adsh) RNA group, which consisted of NRCMs cultured in normoxic condition following AdshRNA transfection; ii) control + adenoviral vectors encoding PHLDA3 shRNA (AdshPHLDA3) group, which consisted of NRCMs cultured under normoxic conditions following AdshPHLDA3 transfection; iii) the $\mathrm{H} / \mathrm{R}+$ AdshRNA group, which consisted of NRCMs cultured under H/R conditions following AdshRNA transfection; and iv) H/R + AdshPHLDA3 group, which consisted of NRCMs cultured in H/R conditions following AdshPHLDA3 transfection. The methods for experimental design were performed according to a method widely reported by previous studies $(9,18-23)$. A total of 20 neonatal rats were used in each group.

Additionally, to detect underlying mechanisms associated with the p-PI3K/AKT pathway, NRCMs were pretreated with $10 \mu \mathrm{M}$ LY294002, a p-PI3K/AKT inhibitor, for $30 \mathrm{~min}$ prior to AdshPHLDA3 administration and were subjected to $\mathrm{H} / \mathrm{R}$ intervention as previously described (24). PBS was used as a control. During this experiment, 16 neonatal rats were used for each group. The protocol for each group was repeated $\geq 4$ times.

Adenoviral construction and transduction into cardiomyocytes. AdshPHLDA3 or AdshRNA as a control were provided by Shanghai GeneChem Co., Ltd. and constructed as previously described (24,25). Briefly, AdshPHLDA3 was synthesized and generated using an AdMax system (Microbix Biosystems Inc.). 293T cells (SunBio, Inc.) were used to package and amplify acquired adenoviruses and the final virus concentration was $1 \times 10^{11}$ plaque-forming units.

Furthermore, for viral transfection in the in vitro experiments, NRCMs were seeded at a density of $1 \times 10^{5}$ cells per 
24-well plate were incubated with AdshPHLDA3 or AdshRNA at 50 multiplicities of infection for $2 \mathrm{~h}$ at $37^{\circ} \mathrm{C}$. Following this, the medium was removed and NRCMs were maintained at $37^{\circ} \mathrm{C}$ with complete medium for $48 \mathrm{~h}$.

Cell viability assay. CCK-8 assays were used to measure cell viability in each group as previously described (24). Optical density values were detected at a wavelength of $450 \mathrm{~nm}$ using a microplate reader (Bio-Rad Laboratories, Inc.). Cell viability was evaluated as the percentage relative to the control group.

Measurement of myocardial injury markers. LDH and CK are commonly used as markers for myocardial death following MIRI $(14,25,26)$. Following the indicated treatments, the medium of cultured NRCMs was collected and commercially available biochemical kits (Nanjing Jiancheng Bioengineering Institute) were used according to the manufacturer's protocol to detect levels of LDH and CK. Results are presented as U/1.

Apoptosis detection. Apoptotic NRCMs were assessed by flow cytometry as previously described (24). Following the indicated treatments, NRCMs were maintained in $100 \mu \mathrm{l}$ binding buffer containing $5 \mu \mathrm{l}$ Annexin V-APC and $5 \mu 1$ 7-minoactinomycin D (7-AAD). Subsequently, stained cells were observed using a flow cytometer (Beckman Coulter, Inc.). NRCMs positive for 7-AAD and Annexin V-APC were identified as apoptotic cells.

Reverse transcription-quantitative PCR (RT-qPCR). RT-qPCR was used to detect mRNA levels as previously described $(4,14)$. Total RNA was extracted from harvested NRCMs with TRIzol $^{\circledR}$ reagent (Invitrogen; Thermo Fisher Scientific, Inc.). RT-qPCR was performed using a SYBR-Green Master Mix kit (Thermo Fisher Scientific, Inc.) on the 7500 ABI Prism system (Applied Biosystems; Thermo Fisher Scientific, Inc.). The following thermocycling conditions were used for the qPCR: $2 \mathrm{~min}$ at $50^{\circ} \mathrm{C}, 10 \mathrm{~min}$ at $95^{\circ} \mathrm{C}, 40$ cycles of $95^{\circ} \mathrm{C}$ for $30 \mathrm{sec}$ and $60^{\circ} \mathrm{C}$ for $30 \mathrm{sec}$. mRNA levels of PHLDA3, CHOP, GRP78 and caspase-12 were normalized to $\beta$-actin. The following primer pairs were used for the qPCR: PHLDA3 forward, 5'-CAT CTACTTCACGCTAGTGACCG-3' and reverse, 5'-TCTGGA TGGCCTGTTGATTCT-3'; CHOP forward, 5'-TAGCTTGGC TGACTGAGGAGC-3' and reverse, 5'-CTTCAGCAAGCT GTGCCACT-3'; GRP78 forward, 5'-GATAATCAGCCCACC GTAACAAT-3' and reverse, 5'-GCAAACTTCTCGGCGTCA TT-3'; caspase-12 forward, 5'-CATTGCCAATTCCGACAA AC-3' and reverse 5'-CCTTCCTTCTCCATCACTGGA-3' and $\beta$-actin forward, 5'-CGTTGACATCCGTAAAGACCTC-3' and reverse, 5'-TAGGAGCCAGGGCAGTAATCT-3'.

Western blotting. Western blotting was performed to detect the protein levels of PHLDA3, PI3K, p-PI3K, AKT, p-AKT, CHOP, GRP78, cleaved caspase-12 and GAPDH in NRCMs after the indicated treatments as previously described $(4,14)$. Briefly, cardiomyocytes were lysed by ice-cold RIPA buffer (Beyotime Institute of Biotechnology). Protein concentrations were detected using a BCA kit (Pierce; Thermo Fisher Scientific, Inc.). Following this, $50 \mu \mathrm{g}$ protein/lane was separated via $10 \%$ SDS-PAGE and transferred onto PVDF membranes (EMD Millipore). PVDF membranes were then incubated with primary antibodies against PHLDA3, PI3K, p-PI3K, AKT, p-AKT, CHOP, GRP78, cleaved caspase-12 and GAPDH overnight at $4^{\circ} \mathrm{C}$. Following three washes with TBS-0.1\% Tween-20, membranes were incubated with horseradish peroxidase-conjugated rabbit anti-rat $\operatorname{IgG}$ secondary antibodies for $2 \mathrm{~h}$ at room temperature. Finally, an enhanced chemiluminescence system (Thermo Fisher Scientific, Inc.) was used to visualize protein bands. Quantity One 1-D software (version 4.6.9; Bio-Rad Laboratories, Inc.) was used for densitometric analysis.

Statistical analysis. SPSS software (version 17.0; SPSS, Inc.) was used for statistical analysis. Data are presented as the mean \pm SD from four independent experiments. Student's t-test or one-way ANOVA followed by Tukey's post hoc test were used for comparisons between groups $(9,22,27)$. $\mathrm{P}<0.05$ was considered to indicate a statistically significant difference.

\section{Results}

PHLDA3 expression is upregulated following MIRI. To explore the possible involvement of PHLDA3 during MIRI, the protein and mRNA levels of PHLDA3 in NRCMs following exposure to $\mathrm{H} / \mathrm{R}$ injury were measured. The results indicated that PHLDA3 protein expression was significantly increased in the $H / R$ group compared with the control group (Fig. 1A). Similarly, the mRNA levels of PHLDA3 in H/R-treated NRCMs was significantly higher compared with the control group (Fig. 1B). These data suggested that PHLDA3 may play a role in MIRI. Furthermore, to detect the effects of PHLDA3 in response to MIRI, AdshPHLDA3 was transfected into NRCMs to inhibit PHLDA3 expression. The results demonstrated that the protein (Fig. 1C) and mRNA (Fig. 1D) levels of PHLDA3 in NRCMs significantly decreased following AdshPHLDA3 transfection compared with the AdshRNA group. Additionally, the mRNA levels of PHLDA3 in H/R-treated NRCMs were measured by RT-qPCR. The results demonstrated that AdshPHLDA3-transfected NRCMs decreased the mRNA levels of PHLDA3 following $H / R$ compared with the H/R + AdshRNA group (data not shown). However, there was no significant difference between the groups (data not shown).

PHLDA3 inhibition represses ERS-induced apoptosis in myocardial $H / R$ injury. To investigate whether PHLDA3 inhibition attenuated H/R-induced myocardial injury, cell viability and LDH/CK release were detected by CCK- 8 assays and ELISA, respectively. The results of the CCK-8 assays (Fig. 2A) demonstrated that cell viability was higher in the Control group relative to the H/R groups. PHLDA3 inhibition following AdshPHLDA3 transfection partially restored the reduced cell viability in response to $H / R$ injury compared with the AdshRNA group. Additionally, H/R-induced LDH (Fig. 2B) and CK (Fig. 2C) release were significantly repressed by PHLDA3 inhibition (AdshPHLDA3 + H/R group vs. AdshRNA + H/R group.

To further investigate the pathological mechanisms of cardioprotective properties following PHLDA3 inhibition and I/R injury, ERS-induced apoptosis was assessed. MIRI is 
A
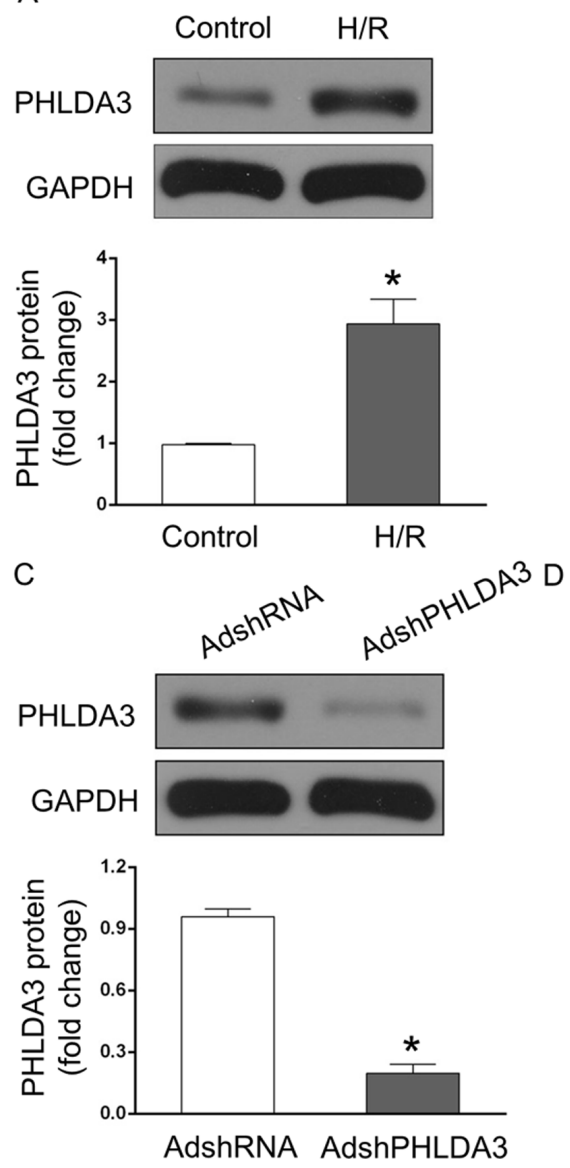

B
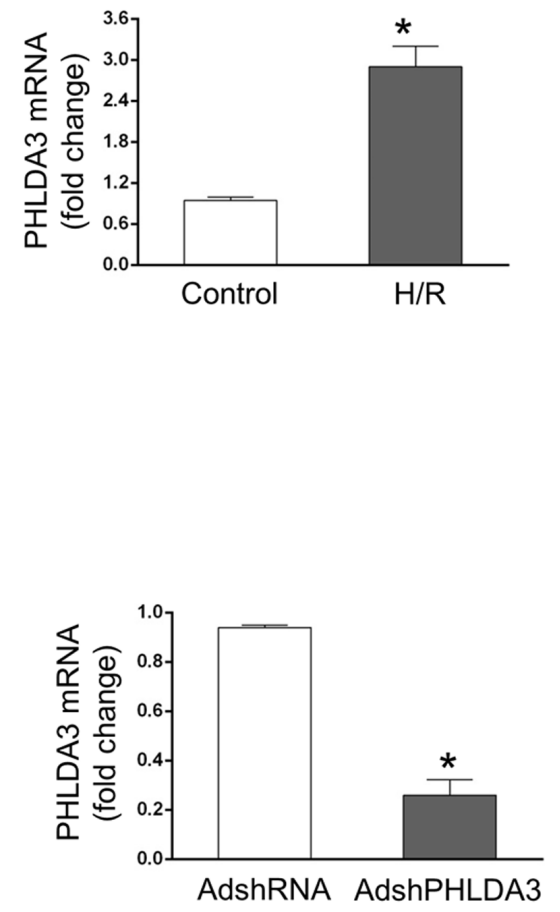

Figure 1. PHLDA3 expression is upregulated following myocardial ischemia/reperfusion injury. (A) PHLDA3 levels in H/R-treated NRCMs were measured by western blotting and quantified bands were normalized to GAPDH. (B) mRNA levels of PHLDA3 in H/R-treated NRCMs were measured by RT-qPCR. (C) Representative bands and quantified data of PHLDA3 levels in adenoviral transfected-NRCMs. (D) mRNA levels of PHLDA3 in AdshRNA/ AdshPHLDA3-transfected NRCMs detected using RT-qPCR. ${ }^{*} \mathrm{P}<0.05$ vs. the control group. Data are presented as the mean \pm standard deviation. $\mathrm{n}=4 /$ group. PHLDA3, pleckstrin homology-like domain family A member 3; H/R, hypoxia/reoxygenation; NRCMs, neonatal rat cardiomyocyte; RT-qPCR, reverse transcription-quantitative PCR; AdshRNA, adenovirus encoding scrambled short hairpin RNA; AdshPHLDA3, adenoviral vectors encoding PHLDA3 shRNA.

associated with the activation of ERS-induced apoptosis $(4,12)$ and PHLDA3 has been demonstrated to be involved in ERS progression (7). To determine whether PHLDA3 impacted myocardial H/R injury in an ERS-associated manner, myocardial apoptotic and ERS-associated proteins, including GRP78, CHOP and cleaved caspase-12, in NRCMs were assessed. As shown in Fig. 2D, low apoptotic rates were observed in the control + AdshRNA and control + AdshPHLDA3 groups; while H/R significantly increased cardiomyocyte apoptosis compared with the control group. AdshPHLDA3 transfection substantially decreased the apoptotic rate following H/R compared with the AdshRNA-treated cells following $\mathrm{H} / \mathrm{R}$ insult. Additionally, the expression levels of myocardial apoptotic and ERS-associated proteins, including CHOP, GRP78 and cleaved caspase-12, were detected by western blotting. As shown in Fig. 2E, H/R significantly increased the protein expression of GRP78, CHOP and cleaved caspase-12 compared with the control group Furthermore, PHLDA3 inhibition significantly decreased the levels of these proteins following $\mathrm{H} / \mathrm{R}$ injury compared with the H/R + AdshRNA group. These results indicated that PHLDA3 inhibition mitigated H/R-induced ERS and subsequent apoptosis in NRCMs.
PHLDA3 inhibition promotes the $p$-PI3K/AKT signaling pathway following $H / R$ injury. The activation of the p-PI3K/AKT pathway is considered as a pro-survival mediator in MIRI $(4,11,28)$. Previous studies demonstrated that PHLDA3-driven alternations of the p-PI3K/AKT pathway participate in multiple pathological processes, including cardiac remodeling and liver injury $(9,13)$. Thus, whether the $\mathrm{p}-\mathrm{PI} 3 \mathrm{~K} / \mathrm{AKT}$ pathway was responsible for the anti-MIRI effects of PHLDA3 inhibition was examined. As shown in Fig. 3, the levels of p-PI3K and p-AKT were significantly reduced following $H / R$ injury compared with normoxic control cells. However, AdshPHLDA3-transfected H/R-treated NRCMs demonstrated significantly increased $\mathrm{p}-\mathrm{PI} 3 \mathrm{~K}$ and p-AKT compared with the AdshRNA-transfected H/R group. Additionally, there were no significant differences in the levels of t-PI3K and t-AKT between the four groups (data not shown). Therefore, these results demonstrated the role of the p-PI3K/AKT pathway in the cardioprotection of PHLDA3 in $\mathrm{H} / \mathrm{R}$ injury.

Blockage of the $p$-PI3K/AKT pathway inhibits the protective effects of PHLDA3 inhibition following $H / R$ injury. Based on the aforementioned results, a rescue study was performed. 

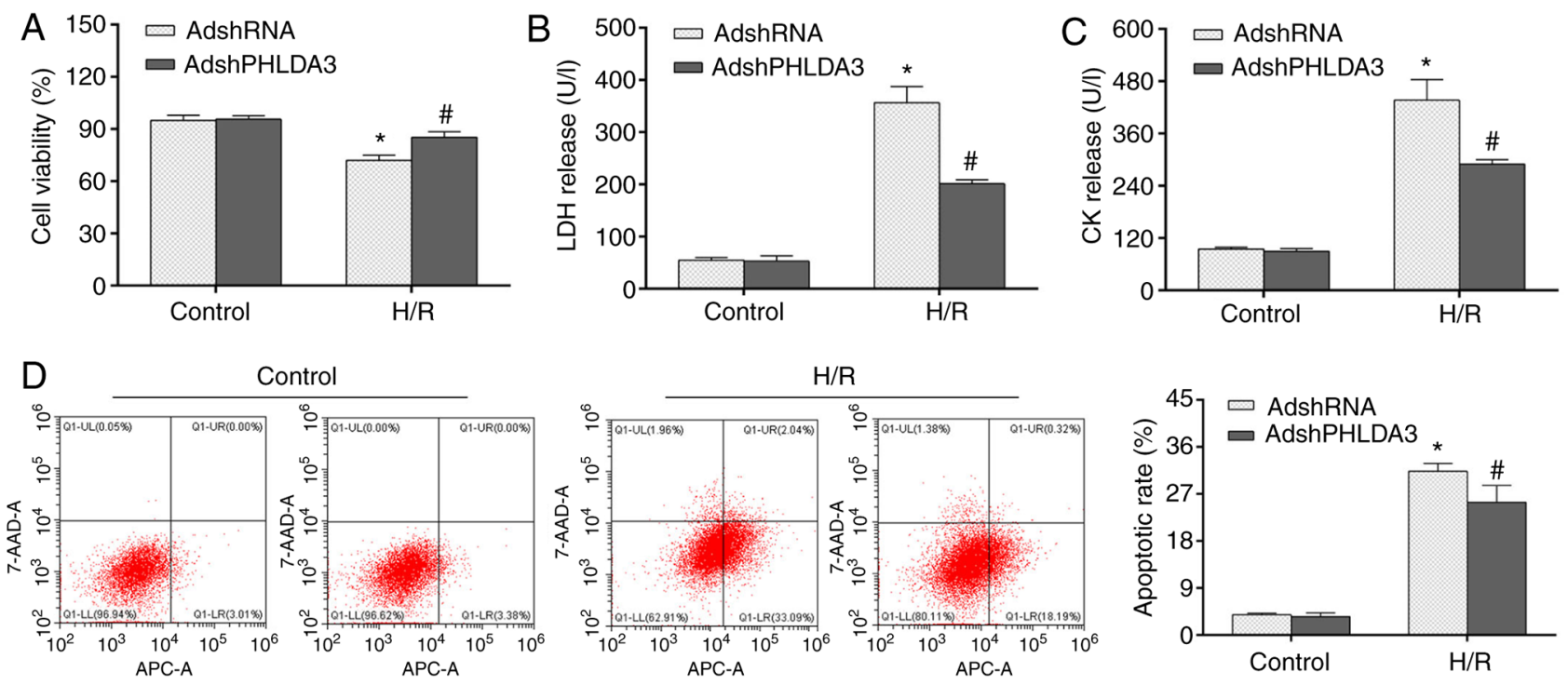

Control
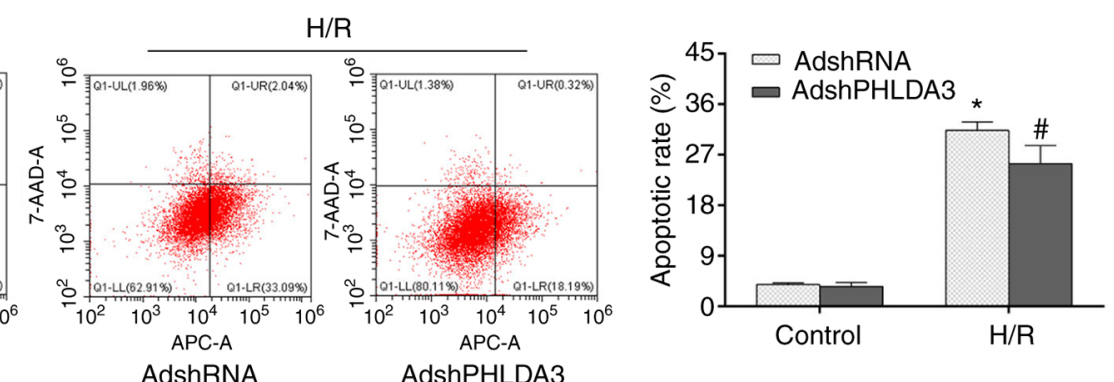

E

AdshRNA

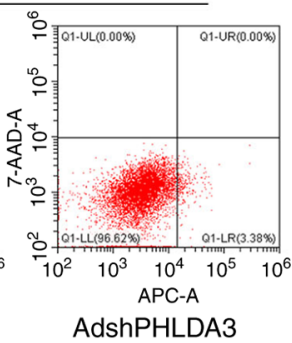

AdshRNA

AdshPHLDA3
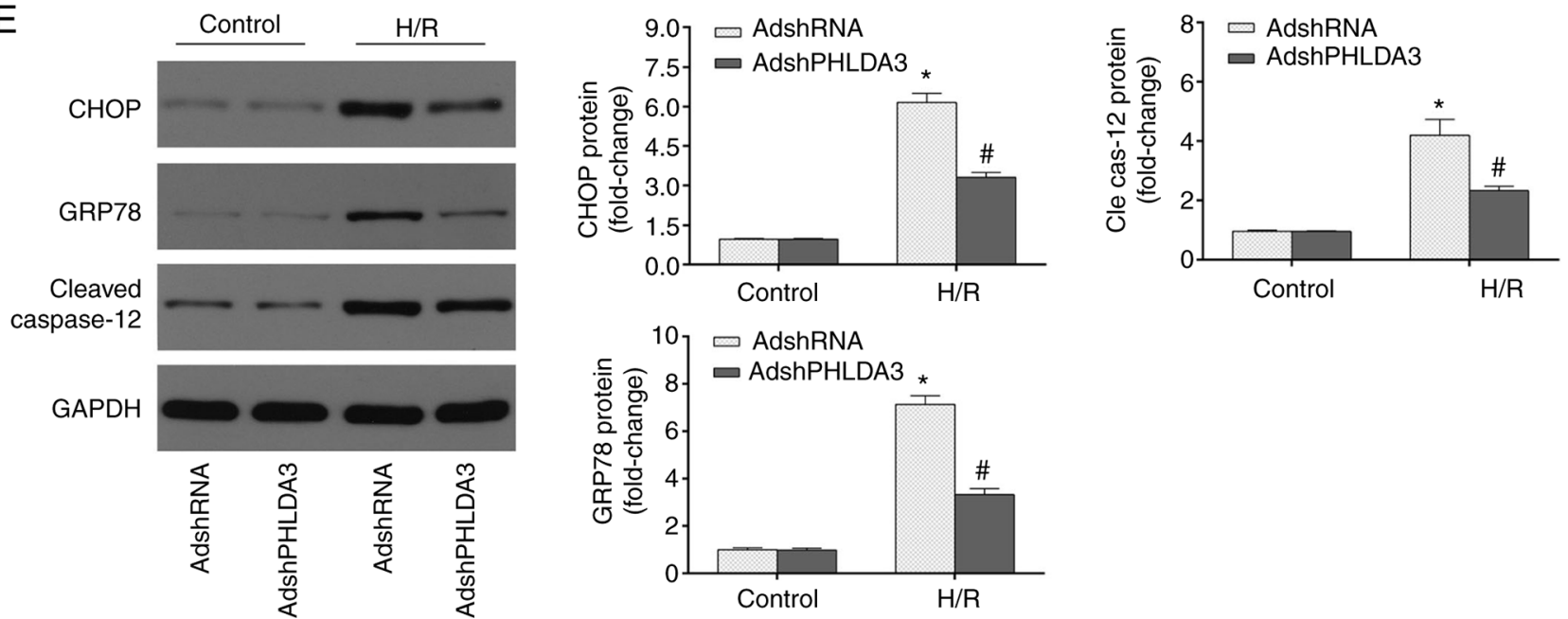

Figure 2. PHLDA3 inhibition alleviates ERS-induced apoptosis during H/R injury. (A) Cell viability was assessed using Cell Counting Kit-8 assays following $\mathrm{H} / \mathrm{R}$ injury. The release of (B) LDH and (C) CK following H/R was detected by ELISA. (D) Apoptotic cells were measured by flow cytometry. (E) Expression of ERS-associated proteins, including GRP78, CHOP and cleaved caspase-12 was detected using western blotting. The left panel shows representative blots and the right panel shows the quantitative results. Data are presented as the mean \pm standard deviation. $n=4$ /group. ${ }^{2} \mathrm{P}<0.05$ vs. AdshRNA or AdshPHLDA3 + control. ${ }^{\text {"P}} \mathrm{P}<0.05$ vs. AdshRNA + H/R group. PHLDA3, pleckstrin homology-like domain family A member 3; ERS, endoplasmic reticulum stress; H/R, hypoxia/ reoxygenation; LDH, lactate dehydrogenase; GRP78, 78 kDa glucose-regulated protein; AdshRNA, adenovirus encoding scrambled short hairpin RNA; AdshPHLDA3, adenoviral vectors encoding PHLDA3 shRNA; 7-AAD, 7-aminoactinomycin D; Cle cas-12, cleaved caspase-12; CK, creatine kinase.

Adenovirus-transfected and H/R-treated cells were treated with the p-PI3K inhibitor LY294002. Cell viability (Fig. 4A), LDH release (Fig. 4B), apoptosis (Fig. 4C) and the mRNA expression of ERS-associated mediators (Fig. 4D-F) were significantly different between the AdshRNA and AdshPHLDA3 groups under H/R + PBS conditions. Treatment with LY294002 inhibited the protective effects of PHLDA3 inhibition on AdshPHLDA3-transfected cell survival compared with the $\mathrm{H} / \mathrm{R}+\mathrm{PBS}+$ AdshPHLDA3-treated NRCMs (Fig. 4A and B). Similar results were observed for apoptosis rates (Fig. 4C) and expression ERS-associated molecules (Fig. 4D-F) following LY294002 treatment. LY294002 administration abolished the protective effects of PHLDA3 inhibition on apoptosis and ERS-associated mediators compared with $\mathrm{H} / \mathrm{R}+\mathrm{PBS}+$ AdshPHLDA3-treated NRCMs. Overall, these results indicated that the cardioprotective contributions of
PHLDA3 inhibition following H/R may be dependent on the p-PI3K/AKT pathway activation and subsequent decrease in expression of ERS-associated apoptosis.

\section{Discussion}

Prior studies have demonstrated that PHLDA3 alleviated pressure overload-induced cardiac remodeling (9) and impaired hemangioblast specification and vascular development (6). However, the role of PHLDA3 and possible molecular mechanisms in MIRI have not been elucidated. The current study demonstrated that PHLDA3 expression was upregulated in cardiomyocytes following H/R injury. Adenovirus-mediated PHLDA3 inhibition, however, reduced myocardial H/R injury, which was characterized by the increase of cell activity and the decreased release of LDH/CK. Additionally, PHLDA3 

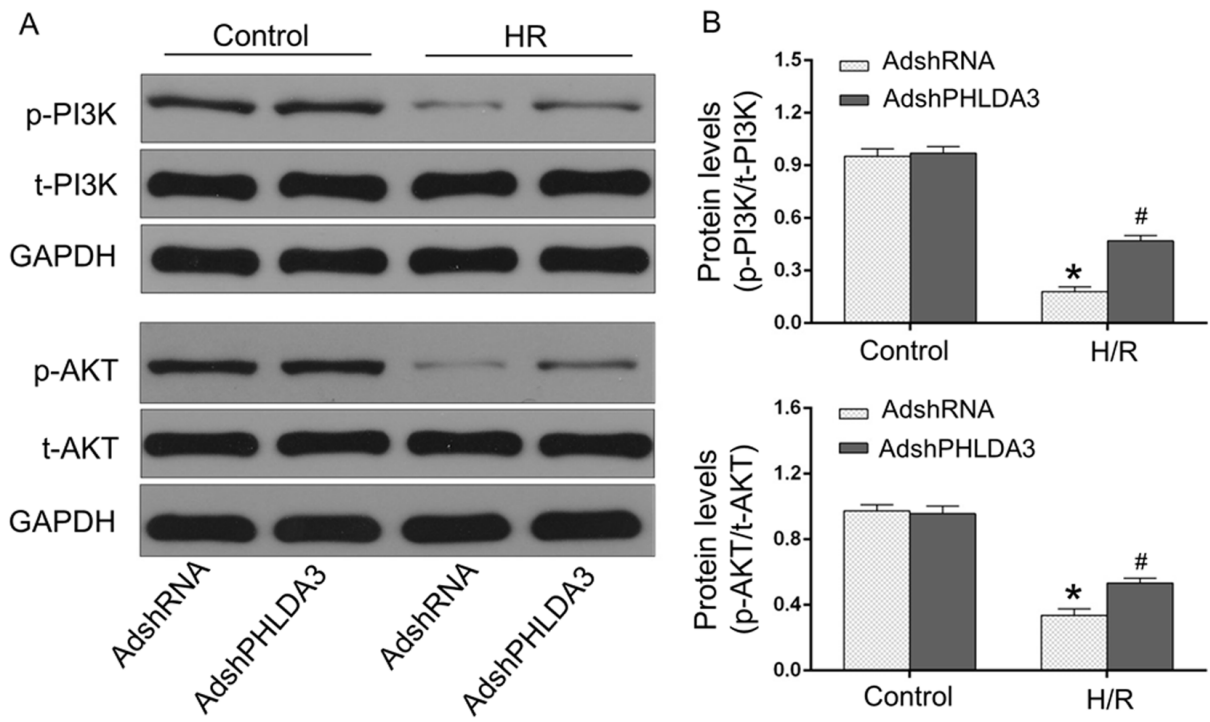

Figure 3. PHLDA3 inhibition activates the p-PI3K/AKT signaling pathway following H/R injury. (A) Representative western blotting images for p-PI3K, t-PI3K, p-AKT and t-AKT. (B) The histogram demonstrates the statistical measurements of the relative intensities normalized to GAPDH. Data are presented the mean \pm standard deviation. $\mathrm{n}=4$ /group. ${ }^{*} \mathrm{P}<0.05$ vs. AdshRNA or AdshPHLDA3 + control. ${ }^{*} \mathrm{P}<0.05$ vs. AdshRNA+ H/R group. PHLDA3, pleckstrin homology-like domain family A member 3; H/R, hypoxia/reoxygenation; AdshRNA, adenovirus encoding scrambled short hairpin RNA; AdshPHLDA3, adenoviral vectors encoding PHLDA3 shRNA; $p$, phosphorylated; $t$, total.
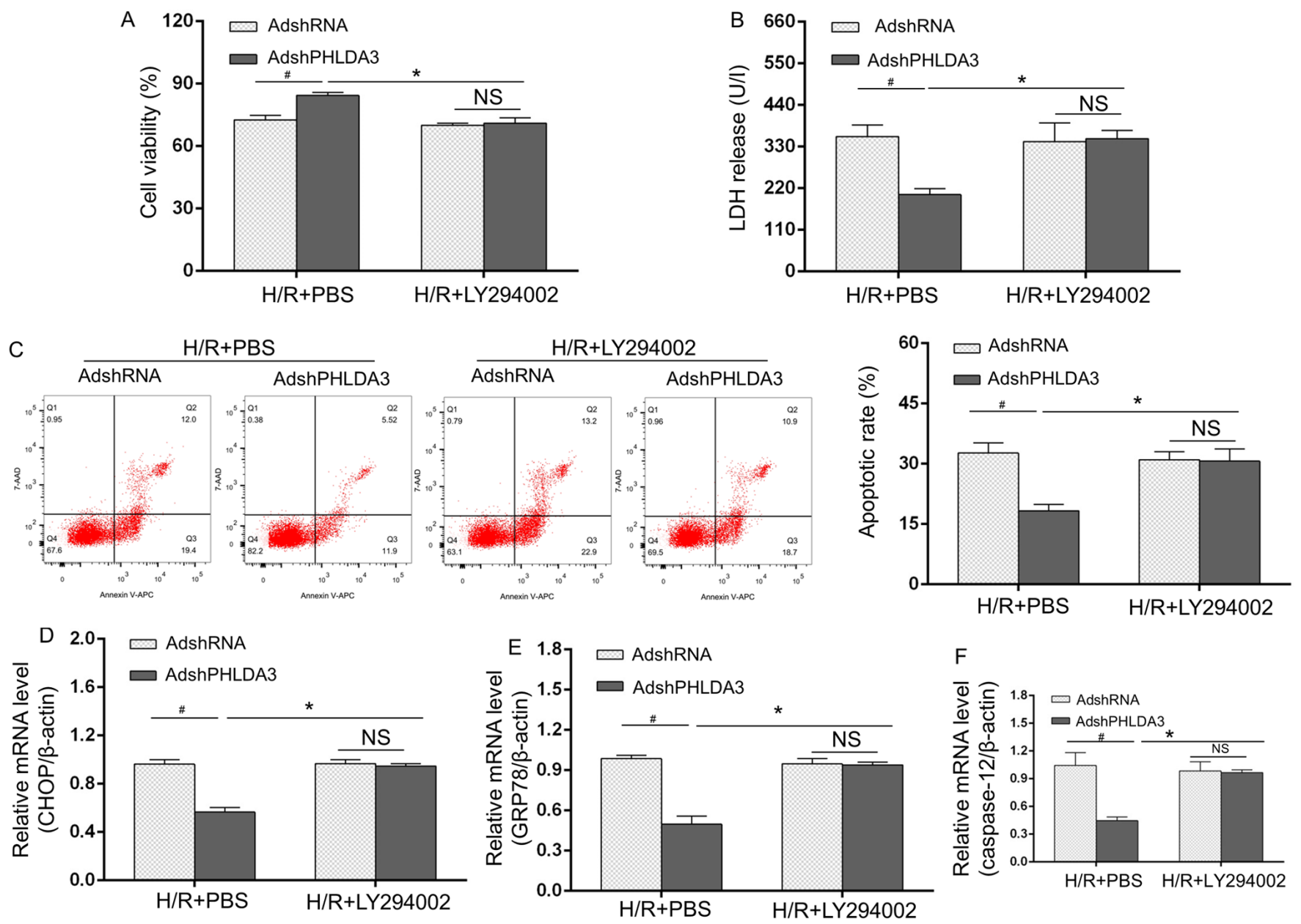

Figure 4. Blockage of the p-PI3K/AKT pathway inhibits the protective effects of PHLDA3 inhibition following H/R injury. (A) Cell Counting Kit-8 assays were used to evaluate cell viability. (B) LDH release was detected by ELISA. (C) Apoptotic rate in each group. mRNA levels of ERS markers, including (D) CHOP, (E) GRP78 and (F) caspase-12, were measured by reverse transcription-quantitative PCR. Data are presented as the mean \pm standard deviation. $\mathrm{n}=4 /$ group. ${ }^{\text {*P }}<0.05$ vs. H/R + AdshPHLDA3 + PBS. ${ }^{*} \mathrm{P}<0.05$ vs. H/R + AdshRNA + PBS. PHLDA3, pleckstrin homology-like domain family A member 3; H/R, hypoxia/ reoxygenation; LDF, lactate dehydrogenase; GRP78, 78 kDa glucose-regulated protein; AdshRNA, adenovirus encoding scrambled short hairpin RNA; AdshPHLDA3, adenoviral vectors encoding PHLDA3 shRNA; NS, not significant. 
inhibition ameliorated cardiomyocyte apoptosis and inhibited the protein expression of ERS-associated molecules, including GRP78, CHOP and cleaved caspase-12. The mechanistic study observed that PHLDA3 inhibition promoted the p-PI3K/AKT signaling pathway, while LY294002, a p-PI3K/AKT inhibitor, reversed its protective roles against $\mathrm{H} / \mathrm{R}$-induced damage and ERS-associated apoptosis. These results indicated that PHLDA3 inhibition exhibited a significant role in ameliorating $\mathrm{H} / \mathrm{R}$ injury and $\mathrm{ERS}$-associated apoptosis mainly via the $\mathrm{p}-\mathrm{PI} 3 \mathrm{~K} / \mathrm{AKT}$-dependent pathway.

Extensive studies have indicated that PHLDA3 exhibits pleiotropic effects in a variety of pathological conditions, including liver injury (7), renal tubular cell death (29) and tumor initiation (8). The underlying pathogeneses are closely associated with apoptosis-regulatory activity $(7,13)$. Notably, recent studies have revealed that PHLDA3 serves important roles in cardiovascular diseases $(6,9)$. For example, in in vivo and in vitro models of pressure overload-induced cardiac remodeling, Liu et al (9) demonstrated that PHLDA3 overexpression acted as a potent therapeutic agent for the protection against hypertrophy and fibrosis. Wang et al (6) indicated that PHLDA3 upregulation affected the specification of hemangioblasts and vascular development. However, whether PHLDA3 participates in the development of MIRI remains unclear and the potential pathological mechanisms are unknown. Notably, a previous study demonstrated that PHLDA3 was a crucial mediator for the progression of ERS and apoptotic response (7). Han et al demonstrated that PHLDA3 overexpression enhanced the levels of ERS markers and facilitated ERS progression, by which it acted as a possible therapeutic agent in limiting ERS-associated hepatocyte apoptosis and cell death (7). In terms of the possible involvement of PHLDA3 in ERS-associated apoptosis, it was hypothesized that PHLDA3 may exert protective effects on cardiomyocytes and serve an important role in ERS-associated apoptosis following H/R insult. The results of the current study provided evidence that PHLDA3 inhibition had an advantageous role in H/R-injured NRCMs and that it reduced H/R-induced and ERS-associated apoptosis.

The PHLDA family of genes consists of three members: PHLDA1, PHLDA2 and PHLDA3 $(13,30)$. The present study and another previous investigation (9) have revealed that PHLDA3 served important roles in cardiovascular diseases (13). Notably, the contributions of other members of PHLDA family have also been demonstrated in cardiovascular insult. For instance, Guo et al (30) demonstrated that PHLDA1 knockdown improved and PHLDA1 overexpression enhanced oxidative stress-induced cardiac injury in MIRI. However, further studies are required to explore whether PHLDA2 participates in cardiovascular diseases, particularly in MIRI.

Timely and effective reperfusion of the ischemic heart has been confirmed to be the most efficacious treatment for acute myocardial infarction $(4,14)$. Nevertheless, the accompanying I/R injury can strongly offset this beneficial effect $(4,14)$. The ERS-elicited apoptotic response is considered to be one of the most critical mechanisms of MIRI $(5,11)$. Previous studies have reported that MIRI results in severe ERS due to markedly enhanced GRP78 levels following MIRI, which subsequently leads to the activation of caspase-12 and
CHOP, eliciting myocardial apoptosis $(5,11)$. The present study demonstrated that MIRI markedly induced myocardial death, apoptosis and ERS. However, PHLDA3 inhibition reduced the levels of ERS-associated proteins including GRP78, CHOP and caspase-12, and ameliorated the parameters associated with the severity of $H / R$ injury. Therefore, PHLDA3 inhibition protected cardiomyocytes against H/R damage, possibly by mitigating ERS-associated apoptosis.

Based on the aforementioned findings, the potential molecular mechanisms by which PHLDA3 inhibition exerts protective effects against H/R injury and ERS-associated apoptosis was investigated. It is well known that p-PI3K and its downstream target serine/threonine kinase $\mathrm{p}-\mathrm{AKT}$ serve critical roles in protecting against MIRI and that the activation of this pathway inhibits ERS-associated myocardial apoptosis $(4,11)$. Importantly, previous studies have demonstrated the close association between PHLDA3 and the p-PI3K/AKT signaling pathway $(6,13)$. For instance, Wang et al (6) demonstrated that PHLDA3 overexpression inhibited the activation of $\mathrm{p}-\mathrm{AKT}$ in vascular development. P-AKT was elevated in PHLDA3-deficient islets following early islet transplantation (13). Moreover, PHLDA3 was reported to repress p-AKT and p-AKT-regulated biological processes in pancreatic endocrine tissue (8). Consistently, the current study confirmed that PHLDA3 inhibition ameliorated H/R-induced apoptosis and increased p-PI3K and p-AKT levels. To further verify the association between the $\mathrm{p}-\mathrm{PI} 3 \mathrm{~K} / \mathrm{AKT}$ pathway and PHLDA3, a specific inhibitor of p-PI3K/AKT, LY294002, was added before H/R. Notably, the cardioprotective efficacy and inhibitory effects on ERS-related apoptosis following PHLDA3 inhibition were abolished in the presence of LY294002. Therefore, these results indicated that PHLDA3 inhibition decreased ERS-associated apoptosis and H/R-induced cell damage in a $\mathrm{p}$-PI3K/AKT-dependent manner. To the best of our knowledge, other molecular mechanisms resulting from PHLDA3 in MIRI have not been reported. A previous study investigated the effect of PHLDA3 on the regulation of the inositol requiring enzyme/X-box binding protein 1 pathway and demonstrated PHLDA3 be involved in liver injury (7). Further studies are required to detect other molecular mechanisms of PHLDA3 apart from the p-PI3K/AKT pathway in MIRI.

In summary, the present study demonstrated that the downregulation of PHLDA3 alleviated MIRI by attenuating ERS-induced apoptosis in a p-PI3K/AKT-dependent manner. Moreover, numerous other signaling pathways are involved in the pathophysiological processes of MIRI (31-33). Further studies are necessary to explore whether other signaling pathways are involved in the effect of PHLDA3 in MIRI. Moreover, the present study reported the effects of PHLDA3 in $\mathrm{H} / \mathrm{R}$-injured NRCMs in an in vitro study. Further investigations will study the effects of PHLDA3 on myocardial histological morphology in I/R-treated rat hearts in vivo. In conclusion, the present results indicated that PHLDA3 may be a prospective therapeutic option for MIRI.

\section{Acknowledgements}

Not applicable. 


\section{Funding}

No funding was received.

\section{Availability of data and materials}

The datasets used and/or analyzed during the current study are available from the corresponding author on reasonable request.

\section{Authors' contributions}

KL, YC and FA conceived and designed the experiments. YQL, KZ and WTZ made substantial contributions to the acquisition of data and manuscript revision, and perfomed the statistical analysis. All authors contributed to the preparation and revision of the manuscript. All authors read and approved the final manuscript.

\section{Ethics approval and consent to participate}

Experiments and animal care were performed in adherence with the Guide for the Care and Use of Laboratory Animals published by the US National Institutes of Health (NIH; 8th Edition; 2011) (17) and were approved by the Hainan Affiliated Hospital of Hainan Medical University (Haikou, China).

\section{Patient consent for publication}

Not applicable.

\section{Competing interests}

The authors declare that they have no competing interests.

\section{References}

1. Dai SH, Wu QC, Zhu RR, Wan XM and Zhou XL: Notch1 protects against myocardial Ischaemia-reperfusion injury via regulating mitochondrial fusion and function. J Cell Mol Med 24: $3183-3191,2020$

2. Satomi S, Morio A, Miyoshi H, Nakamura R, Tsutsumi R, Sakaue H, Yasuda T, Saeki N and Tsutsumi YM: Branched-chain amino acids-induced cardiac protection against ischemia/reperfusion injury. Life Sci 245: 117368, 2020.

3. Kohler D, Granja T, Volz J, Koeppen M, Langer HF, Hansmann G, Legchenko E, Geisler T, Bakchoul T, Eggstein C, et al: Red blood cell-derived semaphorin $7 \mathrm{~A}$ promotes thrombo-inflammation in myocardial ischemia-reperfusion injury through platelet GPIb. Nat Commun 11: 1315, 2020.

4. Zhang BF, Jiang H, Chen J, Guo X, Li Y, Hu Q and Yang S: Nobiletin ameliorates myocardial ischemia and reperfusion injury by attenuating endoplasmic reticulum stress-associated apoptosis through regulation of the PI3K/AKT signal pathway. Int Immunopharmacol 73: 98-107, 2019.

5. Gao J, Guo Y, Liu Y, Yan J, Zhou J, An X and Su P: Protective effect of FBXL10 in myocardial ischemia reperfusion injury via inhibiting endoplasmic reticulum stress. Respir Med 161: $105852,2020$.

6. Wang X, Li J, Yang Z, Wang L, Li L, Deng W, Zhou J, Wang L, Xu C, Chen Q and Wang QK: Phlda3 overexpression impairs specification of hemangioblasts and vascular development. Febs J 285: 4071-4081, 2018.

7. Han CY, Lim SW, Koo JH, Kim W and Kim SG: PHLDA3 overexpression in hepatocytes by endoplasmic reticulum stress via IRE1-Xbp1s pathway expedites liver injury. Gut 65: 1377-1388, 2016.
8. Ohki R, Saito K, Chen Y, Kawase T, Hiraoka N, Saigawa R, Minegishi M, Aita Y, Yanai G, Shimizu H, et al: PHLDA3 is a novel tumor suppressor of pancreatic neuroendocrine tumors. Proc Natl Acad Sci USA 111: E2404-E2413, 2014.

9. Liu J, Liu X, Hui X, Cai L, Li X, Yang Y, Shu S, Wang F, Xia H and Li S: Novel role for pleckstrin homology-like domain family a, member 3 in the regulation of pathological cardiac hypertrophy. J Am Heart Assoc 8: e11830, 2019.

10. Kawase T, Ohki R, Shibata T, Tsutsumi S, Kamimura N, Inazawa J, Ohta $\mathrm{T}$, Ichikawa $\mathrm{H}$, Aburatani $\mathrm{H}$, Tashiro $\mathrm{F}$ and Taya Y: PH domain-only protein PHLDA3 is a p53-regulated repressor of Akt. Cell 136: 535-550, 2009.

11. Shen D, Chen R, Zhang L, Rao Z, Ruan Y, Li L, Chu M and Zhang Y: Sulodexide attenuates endoplasmic reticulum stress induced by myocardial ischaemia/reperfusion by activating the PI3K/Akt pathway. J Cell Mol Med 23: 5063-5075, 2019.

12. Yu L, Li B, Zhang M, Jin Z, Duan W, Zhao G, Yang Y, Liu Z, Chen W, Wang S, et al: Melatonin reduces PERK-eIF2alpha-ATF4-mediated endoplasmic reticulum stress during myocardial ischemia-reperfusion injury: Role of RISK and SAFE pathways interaction. Apoptosis 21: 809-824, 2016.

13. Sakata N, Yamaguchi Y, Chen Y, Shimoda M, Yoshimatsu G, Unno M, Sumi S and Ohki R: Pleckstrin homology-like domain family a, member 3 (PHLDA3) deficiency improves islets engraftment through the suppression of hypoxic damage. PLoS One 12: e187927, 2017.

14. Yang G, Zhang X, Weng X, Liang P, Dai X, Zeng S, Xu H, Huan H, Fang M, Li Y, et al: SUV39H1 mediated SIRT1 trans-repression contributes to cardiac ischemia-reperfusion injury. Basic Res Cardiol 112: 22, 2017.

15. Zhang L, Li C, Zhu Q, Li N and Zhou H: Liraglutide, a glucagon-like peptide-1 analog, inhibits high glucose-induced oxidative stress and apoptosis in neonatal rat cardiomyocytes. Exp Ther Med 17: 3734-3740, 2019.

16. Li L, Sha Z, Wang Y, Yang D, Li J, Duan Z, Wang H and Li Y: Pre-treatment with a combination of Shenmai and Danshen injection protects cardiomyocytes against hypoxia/reoxygenation- and $\mathrm{H} 2 \mathrm{O} 2$-induced injury by inhibiting mitochondrial permeability transition pore opening. Exp Ther Med 17: 4643-4652, 2019.

17. Chen L, Huang J, Ji Y, Zhang X, Wang P, Deng K, Jiang X, $\mathrm{Ma} \mathrm{G}$ and $\mathrm{Li} \mathrm{H}$ : Tripartite motif 32 prevents pathological cardiac hypertrophy. Clin Sci (Lond) 130: 813-828, 2016.

18. Wu G, Liu Y, Huang H, Tang Y, Liu W, Mei Y, Wan N, Liu X and Huang C: SH2B1 is critical for the regulation of cardiac remodelling in response to pressure overload. Cardiovasc Res 107: 203-215, 2015.

19. Ye P, Xiang M, Liao H, Liu J, Luo H, Wang Y, Huang L, Chen M and Xia J: Dual-Specificity phosphatase 9 protects against nonalcoholic fatty liver disease in mice through ASK1 suppression. Hepatology 69: 76-93, 2019.

20. Xiang M, Luo H, Wu J, Ren L, Ding X, Wu C, Chen J, Chen S, Zhang $\mathrm{H}, \mathrm{Yu} \mathrm{L}$, et al: ADAM23 in cardiomyocyte inhibits cardiac hypertrophy by targeting FAK-AKT signaling. J Am Heart Assoc 7: e8604, 2018.

21. Liu R, van Berlo JH, York AJ, Vagnozzi RJ, Maillet $M$ and Molkentin JD: DUSP8 regulates cardiac ventricular remodeling by altering ERK1/2 signaling. Circ Res 119: 249-260, 2016.

22. Xin G, Xu-Yong L, Shan H, Gang W, Zhen C, Ji-Jun L, Ping Y and Man-Hua C: SH2B1 protects cardiomyocytes from ischemia/reperfusion injury via the activation of the PI3K/AKT pathway. Int Immunopharmacol 83: 105910, 2020.

23. Zhang BF, Jiang H, Chen J, Hu Q, Yang S, Liu XP and Liu G: LncRNA H19 ameliorates myocardial infarction-induced myocardial injury and maladaptive cardiac remodelling by regulating KDM3A. J Cell Mol Med 24: 1099-1115, 2020.

24. Sun Y, Liu L, Yuan J, Sun Q, Wang N and Wang Y: RP105 protects PC12 cells from oxygenglucose deprivation/reoxygenation injury via activation of the PI3K/AKT signaling pathway. Int J Mol Med 41: 3081-3089, 2018.

25. Guo X, Jiang H, Yang J, Chen J, Yang J, Ding JW, Li S, Wu H and Ding HS: Radioprotective $105 \mathrm{kDa}$ protein attenuates ischemia/reperfusion-induced myocardial apoptosis and autophagy by inhibiting the activation of the TLR4/NF-NF- $\kappa \mathrm{B}$ signaling pathway in rats. Int J Mol Med 38: 885-893, 2016.

26. Hu X, Zhang K, Chen Z, Jiang H and Xu W: The HMGB1IL17A axis contributes to hypoxia/reoxygenation injury via regulation of cardiomyocyte apoptosis and autophagy. Mol Med Rep 17: 336-341, 2018. 
27. Wang HB, Duan MX, Xu M, Huang SH, Yang J, Yang J, Liu LB, Huang R, Wan CX, Ma ZG, et al: Cordycepin ameliorates cardiac hypertrophy via activating the AMPK $\alpha$ pathway. J Cell Mol Med 23: 5715-5727, 2019.

28. Zhou YH, Han QF, Gao L, Sun Y, Tang ZW, Wang M, Wang W and Yao HC: HMGB1 protects the heart against ischemia-reperfusion injury via PI3K/AkT pathway-mediated upregulation of VEGF expression. Front Physiol 10: 1595, 2019.

29. Lee CG, Kang YJ, Kim HS, Moon A and Kim SG: Phlda3, a urine-detectable protein, causes p53 accumulation in renal tubular cells injured by cisplatin. Cell Biol Toxicol 31: 121-130, 2015.

30. Guo Y, Jia P, Chen Y, Yu H, Xin X, Bao Y, Yang H, Wu N, Sun Y and Jia D: PHLDA1 is a new therapeutic target of oxidative stress and ischemia reperfusion-induced myocardial injury. Life Sci 245: 117347, 2020.
31. Yang J, Yang C, Yang J, Ding J, Li X, Yu Q, Guo X, Fan Z and Wang H: RP105 alleviates myocardial ischemia reperfusion injury via inhibiting TLR4/TRIF signaling pathways. Int J Mol Med 41: 3287-3295, 2018

32. Kong T, Liu M, Ji B, Bai B, Cheng B and Wang C: Role of the extracellular signal-regulated kinase $1 / 2$ signaling pathway in ischemia-reperfusion injury. Front Physiol 10: 1038, 2019.

33. Huang CK, Kafert-Kasting S and Thum T: Preclinical and clinical development of noncoding RNA therapeutics for cardiovascular disease. Circ Res 126: 663-678, 2020. Attribution-NonCommercial-NoDerivatives 4.0 International (CC BY-NC-ND 4.0) License. 